\title{
Mindfulness-based cognitive therapy $v$. group psychoeducation for people with generalised anxiety disorder: randomised controlled trial
}

Samuel Yeung Shan Wong, Benjamin Hon Kei Yip, Winnie Wing Sze Mak, Stewart Mercer, Eliza Yee Lai Cheung, Candy Yuet Man Ling, Wacy Wai Sze Lui, Wai Kwong Tang, Herman Hay Ming Lo, Justin Che Yuen Wu, Tatia Mei Chun Lee, Ting Gao, Sian M. Griffiths, Peter Hoi Sing Chan and Helen Shuk Wah Ma

\section{Background}

Research suggests that an 8-week mindfulness-based cognitive therapy (MBCT) course may be effective for generalised anxiety disorder (GAD).

\section{Aims \\ To compare changes in anxiety levels among participants with GAD randomly assigned to $\mathrm{MBCT}$, cognitive-behavioura therapy-based psychoeducation and usual care.}

\section{Method}

In total, 182 participants with GAD were recruited (trial registration number: CUHK_CCT00267) and assigned to the three groups and followed for 5 months after baseline assessment with the two intervention groups followed for an additional 6 months. Primary outcomes were anxiety and worry levels.

\section{Results}

Linear mixed models demonstrated significant group $\times$ time interaction $(F(4,148)=5.10, P=0.001)$ effects for decreased anxiety for both the intervention groups relative to usual care. Significant group $\times$ time interaction effects were observed for worry and depressive symptoms and mental health-related quality of life for the psychoeducation group only.

\section{Conclusions}

These results suggest that both of the interventions appear to be superior to usual care for the reduction of anxiety symptoms.

\section{Declaration of interest}

None.

\section{Copyright and usage}

(c) The Royal college of Psychiatrists 2016.
Generalised anxiety disorder (GAD) is a chronic psychiatric disorder characterised by pervasive, persistent and uncontrollable worry. ${ }^{1}$ It is associated with significant functional impairment, ${ }^{2}$ morbidity and healthcare utilisation. ${ }^{3}$ Although cognitivebehavioural therapy (CBT) has been shown to be effective for treating $\mathrm{GAD},{ }^{4}$ not all those treated are able to achieve a high level of functioning after treatment. ${ }^{5}$ Moreover, there is often a shortage of CBT therapists and the individual therapeutic approach can be expensive in healthcare systems with limited resource. ${ }^{6}$ This calls for the evaluation of other potential treatments such as group interventions that may be more cost-effective. Mindfulness-based interventions have been used and studied for a variety of physical and psychological conditions. ${ }^{7-11}$ Mindfulness-based cognitive therapy (MBCT), a psychological intervention developed by clinical psychologists based on mindfulness-based stress reduction with integration of cognitive-behavioural elements, has been shown to be effective in reducing relapse among people who have recurrent episodes of major depression ${ }^{11-13}$ and is now being incorporated into the guidelines of the UK's National Institute for Health and Care Excellence (NICE) as a treatment option for those who have recurrent depression. ${ }^{12-14}$ Most studies that evaluated the effectiveness of MBCT have been conducted among patients with recurrent depression. A limited number of studies have been conducted to evaluate the effects of MBCT in reducing anxiety and worry among people with GAD. Moreover, these studies have been limited by their study design such as not having a control group for comparison, ${ }^{15-18}$ having a small sample size ${ }^{15-19}$ or no randomisation. ${ }^{19}$ Mindfulness-based stress reduction (MBSR), without the cognitive components, has also been investigated previously, ${ }^{20,21}$ and more recently ${ }^{22}$ a randomised controlled trial (RCT) comparing MBSR with an active control has shown promising results on the reduction of clinically relevant anxiety symptoms among patients with GAD. To our knowledge, the current study is one of the few studies that had included an active comparison group to evaluate the effectiveness of MBCT in reducing anxiety and worry levels among patients with GAD recruited from primary care and the community. We hypothesised that participants in the MBCT group would do better than those in a psychoeducation control group using CBT principles (psychoeducation) and also those in a usual care control group in terms of reducing anxiety symptoms in this population. We included psychoeducation as a comparison group for pragmatic reasons since psychoeducation group therapy using CBT principles has been suggested as a low-intensity intervention for people with GAD in primary care. ${ }^{23}$

\section{Method}

The protocol of this study has been published previously, ${ }^{24}$ although the follow-up assessment for the usual care group ended at 5 months after baseline assessment because of changes in service provision in the local area (before the trial started there was a waiting time of 9-12 months for patients to be seen by a psychiatrist or a psychologist in public clinics, which was reduced to 6 months during the trial with a new service programme). In brief, this RCT (trial registration number: CUHK_CCT00267) included three study arms. There was an MBCT group led by trained instructors, a psychoeducation group using CBT principles led by clinical psychologists and a usual care control group whose participants were offered MBCT at the end of the 5 months after baseline assessment. Self-reported assessments were administered 
at similar time points, including baseline, 2 months after baseline assessment (immediately post-intervention for the treatment arms) and at 5 months after baseline assessment for all three arms. Both the MBCT and psychoeducation group were further followed up at 8 and 11 months after baseline assessment, whereas the usual care group was only followed up to 5 months after baseline assessment. The study was approved by the Clinical Research Ethics Committee (CREC) of the Joint Chinese University of Hong Kong - New Territories East Cluster. The CREC reference number is CRE-2009.353-T.

\section{Participants}

All participants were recruited from: (a) advertisements placed in the health education columns of local newspapers; (b) public general practice or family medicine clinics; and (c) non-governmental organisations and community centres that cater for people with chronic conditions.

All recruited participants fulfilled the following criteria: (a) aged 21-65; (b) with a DSM-IV principal diagnosis ${ }^{1}$ of GAD on a Structured Clinical Interview for DSM-IV (SCID) and a score of 19 or above using the Chinese version of the Beck Anxiety Inventory (BAI) at baseline; ${ }^{25,26}$ (c) could understand Cantonese; (d) were willing to attend either the MBCT or psychoeducation group sessions; (e) if they were on medications for anxiety, they needed to have been on stable doses for at least 2 months before starting the intervention.

Participants were excluded if they: (a) were illiterate as they would have been unable to complete the self-report assessment; (b) had psychiatric and medical comorbidities that were potentially life threatening (i.e. psychosis, suicidal ideation, terminal medical illness) or conditions expected to severely limit patient participation or adherence (such as psychosis, current substance misuse, dementia, pregnancy); (c) were currently seeing a cognitivebehavioural therapist or psychotherapist/counsellor for any psychological problems; and (d) undertook regular meditation or yoga practice (or had previously done so).

All interested participants were screened initially over the telephone, using these inclusion and exclusion criteria, by trained research assistants with a graduate degree in psychology or public health. Those initially screened and deemed eligible were then scheduled a diagnostic interview with the principal investigator to further confirm eligibility using SCID and a structured questionnaire. They were screened for common psychiatric disorders (major depression, somatic symptoms, alcohol dependence and panic disorder) in primary care using the Primary Health Questionnaire (PHQ). ${ }^{27}$ Among participants who screened positive on the GAD schedule of the PHQ, the SCID was further conducted to confirm the GAD diagnosis by the principal investigator. Participants were then informed about their eligibility for the study and were further contacted according to the programme schedule once it was available. Written informed consent was obtained from all participants before taking part in this study and both the principal investigator and the research assistant were trained to use SCID by a psychiatrist.

Following the diagnostic interview, a simple randomisation method was used to randomly assign eligible participants to one of the three groups using the Microsoft Excel RAND function. For every batch of participants recruited, a third of the participants were randomised to the MBCT group, a third to the psychoeducation group and the rest to the control group. To ensure concealment of randomisation, a biostatistician who was not part of this study pre-generated random numbers from a normal distribution. Participants were ranked in order according to their generated values. Participants ranked in the top third of the list were assigned to group $\mathrm{A}$, the middle third to group $\mathrm{B}$ and the remaining ones to group $\mathrm{C}$, where $\mathrm{A}, \mathrm{B}$ and $\mathrm{C}$ represents the treatment the patient will receive (for example $\mathrm{A}, \mathrm{MBCT}$ group; $\mathrm{B}$, psychoeducation group; $\mathrm{C}$, control group) and only the research coordinator could decode it. The timing and venue of the classes for the two intervention groups were arranged to avoid interaction and exchange of information between participants in these two groups.

\section{Treatment arms}

\section{MBCT group}

Five MBCT groups were led by two clinical psychologists and one social worker who were all experienced in leading an MBCT group. All of them had attended intensive MBCT and MBSR training retreats and had both practised and conducted MBCT with patients for at least 2 years. The intervention consisted of weekly sessions for 8 weeks each lasting $2 \mathrm{~h}$ involving up to 15 participants. Our intervention programme followed the MBCT for depression protocol published in the book by Segal et al. ${ }^{12}$ Modifications were made by a team of MBCT instructors in order to make the intervention more suitable for people with anxiety disorders with the cognitive-behavioural components dealing with depression being replaced by components dealing with anxiety. This included discussing the cognitive-behavioural model of GAD in session two, automatic anxiety thoughts in session four, reactive-avoidance and ruminative worrying in session five and the development of an action plan in line with personal values and relapse prevention of anxiety in session seven.

The session summary can be found in online supplement DS1. During the intervention period, participants in this group were given daily homework exercises including guided awareness exercises via audio instruction on compact discs, which included sitting meditation, body scan and mindful movements. Moreover, shorter unguided awareness exercises such as the 3 min breathing space were also included in the homework, with the aim of increasing moment-by-moment awareness of feelings, thoughts and bodily sensations together with exercises designed to integrate the application of mindfulness skills into daily activities. All sessions were audiotaped with a subset reviewed to ensure the fidelity of the programme. All participants were instructed to practise mindfulness meditation for $45 \mathrm{~min}$ a day.

\section{Psychoeducation group}

The psychoeducation intervention was designed to be comparable with MBCT in terms of the course structure and the therapist's contact time and attention, with participants having to comply with an agenda during each session with a similar amount of homework assignments to that of the MBCT group. As for the MBCT group, it consisted of weekly sessions for 8 weeks each lasting $2 \mathrm{~h}$ involving up to 15 participants with didactic teaching and minimal group interaction and discussion. The content of the teaching was based on White's book Treating Anxiety and Stress, a handbook that is used by clinical psychologists to help people cope with anxiety using the cognitive-behavioural approach. ${ }^{28} \mathrm{~A}$ brief description of the schedule of psychoeducation is presented in online supplement DS2. The topics included preparing for stress control, learning about stress, controlling one's body, thoughts and action; controlling one's panic, insomnia, depression and future. In addition to the didactic teaching content, simple relaxation skills such as muscle relaxation skills were also taught during class although instructors were asked, as best as they could, not to teach any skills in a way that may enhance mindfulness. Two clinical psychologists with at least 2 years' experience in CBT practice or teaching were employed to lead the psychoeducation groups. 


\section{Usual care group}

Participants in the usual care (control) group did not receive any specific intervention but they are allowed unrestricted access to primary care services. In Hong Kong, the average consultation time for public primary care clinics is about $6 \mathrm{~min}$ and it is often difficult for doctors to have enough time to deal with patients' emotional problems. The waiting time for referral to be seen by mental health service specialists is at least 6 months.

\section{Outcome measures}

Participants' demographic information including age, gender, marital status, education levels, monthly income, religious beliefs and number of family members were also collected at baseline. All outcome measures and details of health service utilisations were collected at similar time points (baseline, 2 months after baseline assessment and 5 months after baseline assessment) for all three groups. Participants randomised to either of the intervention groups (MBCT or psychoeducation) were further followed for another 6 months and both primary and secondary outcome measures were assessed at 8 and 11 months after baseline assessment.

The primary outcome measures were clinically relevant anxiety symptoms measured by the Chinese version of the BAI and worry symptoms measured by the Penn State Worry Questionnaire (PSWQ) 5 months after baseline assessment $(3$ months postintervention). ${ }^{29-32}$ Secondary outcome measures included: clinically relevant depressive symptoms measured by the validated Chinese version of the Center for Epidemiologic Studies Depression Scale (CES-D), with the cut-off point of 16 being used to indicate 'significant' depressive symptoms; ${ }^{33}$ the validated Chinese version of the Medical Outcomes Study Short-Form Health Survey (SF-12), the Mental Component Summary (MCS-12) and Physical Component Summary (PCS-12), which reported health-related quality of life; ${ }^{34}$ and the Chinese version of the Five Facet Mindfulness Questionnaire (FFMQ). ${ }^{35,36}$ Both primary and secondary outcome measures data were collected at baseline, 2, 5, 8 and 11 months after baseline assessment.

\section{Statistical analysis}

To investigate significant changes over time, linear mixed models (LMM) were conducted for both primary and secondary outcomes following the intention-to-treat principle. A two-sided $P$-value of 0.05 or less was considered to be statistically significant. The use of LMM provided the means to include participants with incomplete data (missed 1 or 2 questionnaires) to assess the treatment effect over time (i.e. trend or group $\times$ time interaction). In our models, intervention group, time and the interactions between the intervention group and time were treated as fixed factors, and an unstructured covariance structure was employed. Statistical analysis of the primary and secondary outcome measures, including BAI, PSWQ, CES-D, SF-12, FFMQ as well as health service utilisation over time were made. In addition, to account for differences in treatment effect as a result of difference in adherence among the two intervention groups, complier-adjusted causal effect (CACE) analysis was conducted according to the causal framework and estimation approaches described previously. ${ }^{37-39}$ The outcome was the change in BAI score from baseline to 5 months after baseline assessment. We defined CACE as the difference in mean BAI score change between the compliers in the two intervention groups and the compliers in the control group. Participants were defined as compliers if they have complied with at least $80 \%$ of classes (i.e. $\geqslant 7$ classes attended), a commonly used cut-off. $^{40,41}$ Treatment specific (MBCT/ psychoeducation) CACE was estimated. Standard errors were obtained by bootstrapping technique. ${ }^{37}$ The CACE analysis was conducted using $\mathrm{R}$, all other analyses were conducted in PASW Statistics 18.

\section{Sample size calculation}

At the time of the trial no studies had compared MBCT with an active control for people with GAD therefore the research findings from a study that compared CBT with an education group were used for sample size calculation. ${ }^{42}$ Assuming a common standard deviation of 7.4 and that the average mean change in BAI score at post-intervention for patients in the MBCT group was 5.6, that in the psychoeducation group it was 2.0 and that in the usual care group it was 1.1 with a type I error of $5 \%$ and $80 \%$ power to detect statistically significant differences between the MBCT and psychoeducation group, as well as MBCT and the usual care group, the required sample size was 53 participants per group. With a presumed drop-out rate of $30 \%$, we aimed to recruit 76 participants per group.

\section{Results}

A total of 1209 potential participants were screened by telephone for eligibility. Among these, 263 participants with anxiety symptoms were scheduled for diagnostic interviews and to confirm their eligibility with the principal investigator. In the end, 182 participants who met the inclusion criteria were randomised. Two participants from the MBCT group and one from the psychoeducation group dropped out before the start of the intervention because of time constraints. Four participants who were not happy with the randomisation results quit the usual care control group immediately without returning the baseline questionnaires. Details of the recruitment process are shown in Fig. 1.

The mean age of our sample was 50 years $($ s.d. $=10)$. The majority of our participants were women (79\%). The demographic characteristics of our participants are presented in Table 1. All participants scored more than 16 (the cut-off threshold for having clinically relevant depressive symptoms) on the CES-D and thus were comorbid with depressive symptoms. A total of 61 participants $(33.5 \%)$ had been taking regular medication for treating GAD and/or depressive symptoms for more than 2 months, with 26 people in the MBCT group (42.6\%), 14 in the psychoeducation group $(23.0 \%)$ and 21 in the usual care group (35.0\%).

The mean number of classes attended was $6.4($ s.d. $=1.9)$ for the MBCT group and 7.1 (s.d.=1.5) for the psychoeducation group. In total, $43(71 \%)$ participants in the MBCT group attended six or more sessions, 24 of whom (39\%) attended all eight sessions. Whereas in the psychoeducation group, 54 (89\%) participants attended six or more sessions and more than half (56\%) completed all eight psychoeducation sessions (see online Table DS1). No significant differences were observed on baseline outcome measures between the completers and the non-completers.

\section{Effects on primary outcome measures}

The BAI scores in both the MBCT and psychoeducation groups decreased significantly at 2 and 5 months after baseline assessment with no change observed for the usual care group. The estimated means and $95 \%$ confidence intervals as generated by the LMM procedure were used to produce the trajectories in Fig. 2(a). At 2 and 5 months after baseline assessment, a significant relative change of score was revealed between the MBCT $v$. usual care 


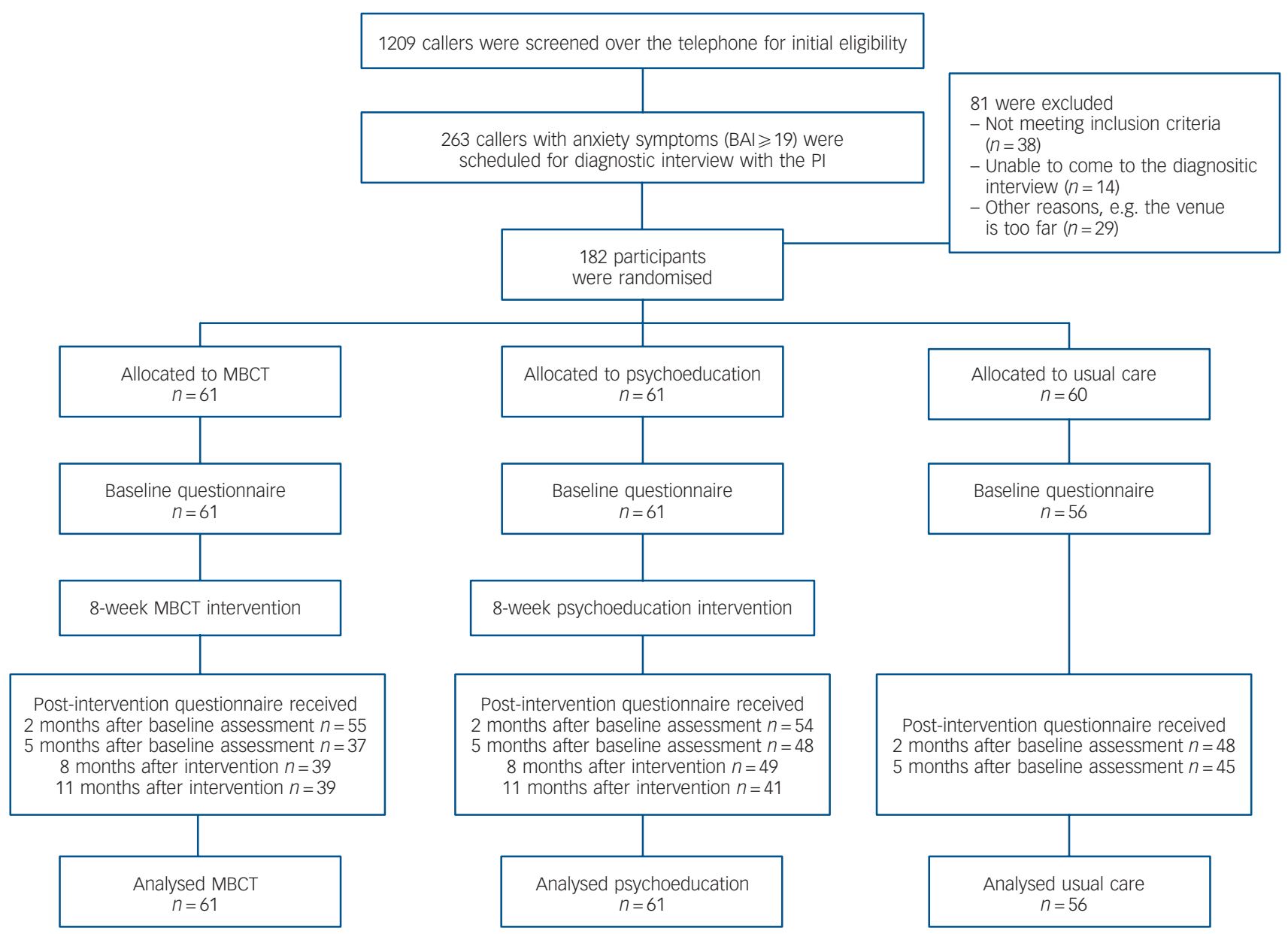

\section{Fig. 1 Participant flow chart.}

BAl, Beck Anxiety Inventory; PI, principal investigator; $\mathrm{MBCT}$, mindfulness-based cognitive therapy.

\begin{tabular}{|c|c|c|c|c|}
\hline & $\begin{array}{c}\text { Total } \\
(n=182)\end{array}$ & $\begin{array}{l}\text { Mindfulness-based cognitive } \\
\text { therapy group }(n=61)\end{array}$ & $\begin{array}{l}\text { Psychoeducation } \\
\text { group }(n=61)\end{array}$ & $\begin{array}{l}\text { Usual care group } \\
\qquad(n=60)\end{array}$ \\
\hline Age, years: mean (s.d.) & $50.00(10.02)$ & $50.40(9.95)$ & 50.79 ( 9.57) & $48.78(10.59)$ \\
\hline Gender, $n$ (\%) & 182 & 61 & 61 & 60 \\
\hline Female & $144(79.1)$ & $48(78.7)$ & $48(78.7)$ & $48(80.0)$ \\
\hline Male & $38(20.9)$ & $13(21.3)$ & $13(21.3)$ & $12(20.0)$ \\
\hline Education, $n$ (\%) & 178 & 59 & 60 & 59 \\
\hline Primary & $28(15.7)$ & $10(16.9)$ & $7(11.7)$ & $11(18.6)$ \\
\hline Secondary & $90(50.6)$ & $31(52.5)$ & $31(51.7)$ & $28(47.5)$ \\
\hline Diploma & $21(11.8)$ & $9(15.3)$ & $6(10.0)$ & $6(10.2)$ \\
\hline Degree or above & $39(21.9)$ & $9(15.3)$ & $16(26.7)$ & $14(23.7)$ \\
\hline Employment, $n(\%)$ & 173 & 58 & 59 & 56 \\
\hline Unemployed/housewife/retired & $100(57.8)$ & $34(58.6)$ & $33(55.9)$ & $33(58.9)$ \\
\hline Employed & $73(42.2)$ & $24(41.4)$ & $26(44.1)$ & $23(41.1)$ \\
\hline Marital status, $n(\%)$ & 180 & 60 & 60 & 60 \\
\hline Married & $129(71.7)$ & $38(63.3)$ & $48(80.0)$ & $43(71.7)$ \\
\hline Single/separated & $51(28.3)$ & $22(36.7)$ & $12(20.0)$ & $17(28.3)$ \\
\hline Income, HK\$: n (\%) & 154 & 48 & 52 & 54 \\
\hline$<10000$ & $71(46.1)$ & $21(43.8)$ & $22(42.3)$ & $28(51.9)$ \\
\hline $10-20000$ & $43(26.0)$ & $16(33.3)$ & $12(23.1)$ & $15(27.8)$ \\
\hline$>20000$ & $40(26.0)$ & $11(22.9)$ & $18(34.6)$ & $11(20.4)$ \\
\hline Religion, $n(\%)$ & 177 & 60 & 61 & 56 \\
\hline Christianity/Catholicism & $65(36.7)$ & $19(31.7)$ & $27(44.3)$ & $19(33.9)$ \\
\hline Buddhism/Taoism & $18(10.2)$ & $6(10.0)$ & $4(6.6)$ & $8(14.3)$ \\
\hline Non-religious & $94(53.1)$ & $35(58.3)$ & $30(49.2)$ & $29(51.8)$ \\
\hline Number of family members, mean (s.d.) & $3.04(1.18)$ & $3.00(1.21)$ & $3.22(1.31)$ & $2.88(0.96)$ \\
\hline
\end{tabular}


groups and between the psychoeducation $v$. usual care groups (Table 2). No differences were found in BAI scores between the MBCT and psychoeducation groups at any time point. Overall, LMM demonstrated a significant group $\times$ time interaction $(F(4,148)=5.10, P=0.001)$.

For the PSWQ scores in the MBCT, psychoeducation and usual care groups, the estimated means and $95 \%$ confidence intervals as generated by the LMM procedure were used to produce trajectories in Fig. 2(b). At 5 months after baseline assessment, but not at 2 months after the baseline assessment, a significant relative change of score was revealed between the psychoeducation $v$. usual care groups but not between the MBCT $v$. usual care or MBCT $v$. psychoeducation groups (Table 2).

As stated previously, participants in the usual care group were followed up only until 5 months after the baseline assessment. Thus, follow-up data at 8 and 11 months after baseline assessment were only available for the MBCT and the psychoeducation groups. Both BAI and PSWQ scores continued to decrease significantly within the MBCT and psychoeducation groups at 8 and 11 months after baseline assessment (i.e. significant time effect) but there were no significant group differences between the psychoeducation and MBCT groups at these two time points (Fig. 2).

\section{Effects on secondary outcome measures}

Statistical analysis showed a significant group $\times$ time interaction (CES-D: $\quad F(4,54)=3.6, \quad P=0.08 ; \quad$ MCS-12: $\quad F(4,147)=4.5$, $P=0.002)$ in the CES-D and MCS-12 scores. As shown in Figs $3(\mathrm{a})$ and 3(c), significant improvements over time were observed only within the psychoeducation group for the CES-D and MCS-12. Significant group differences were seen for these two scales between the psychoeducation and usual care groups at 2 and 5 months after baseline assessment. However, no significant group differences were observed between the MBCT and usual care groups or the MBCT and psychoeducation groups at these time points. Moreover, there was no significant group difference in these outcomes between the MBCT and psychoeducation groups at 8 and 11 months.

In terms of mindfulness, there was a group $\times$ time interaction $(F(4,148)=3.6, P=0.008)$ for the FFMQ. Scores on the FFMQ in both the MBCT and psychoeducation groups increased significantly at 2 and 5 months after baseline assessment (Fig. 3(d)). The significant change in scores was also reported for the MBCT $v$. usual care and psychoeducation $v$. usual care groups at 2 and 5 months after baseline assessment. No group difference was observed between the MBCT and psychoeducation groups.

Based on the 8 and 11 months after baseline assessment result, both the intervention groups showed significant improvements on the CES-D, PCS-12, MCS-12 and FFMQ scores, however there was no statistically significant difference between the two groups (Fig. 3).

Differences in number of medical visits made per month was only observed at 5 months after baseline assessment between the psychoeducation and the usual care groups $(P=0.025)$, but not between the MBCT $v$. psychoeducation groups or between the MBCT $v$. usual care groups.

\section{CACE analysis}

There was no statistically significant difference in class attendance between the two intervention groups (Table DS1, Fisher exact test $P=0.2147)$ or when we dichotomised patients into compliers or non-compliers $\left(\chi^{2}\right.$-test $\left.P=0.074\right)$. In addition, no baseline covariate was shown to be significantly associated with the complier status. The CACE estimates obtained for MBCT and psychoeducation were $-8.56 \quad($ s.e. $=3.85)$ and -8.73 (s.e. $=2.41)$ respectively.
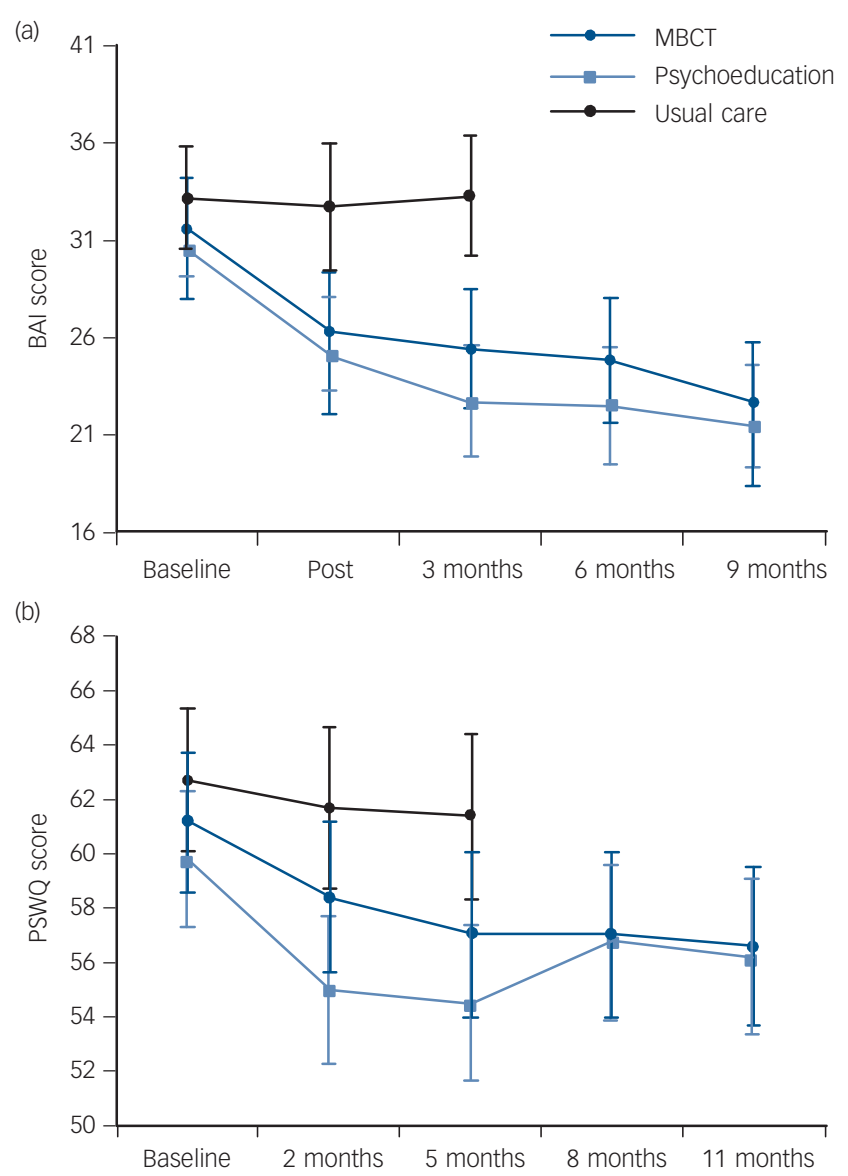

Fig. 2 Estimated mean scores for anxiety symptoms and worry symptoms of participants in the mindfulness-based cognitive therapy (MBCT), psychoeducation and usual care groups over the study period.

(a) Anxiety symptoms as measured by the Beck Anxiety Inventory (BAl) ; (b) worry symptoms as measured by the Penn State Worry Questionnaire (PSWO). Error bars represent the $95 \%$ confidence intervals.

Table 2 Estimated parameters of linear mixed model for Beck Anxiety Inventory and Penn State Worry Questionnaire ${ }^{a}$

\begin{tabular}{lcc} 
Grouptime & $\beta$ (s.e.) & $95 \% \mathrm{Cl}$ \\
Beck Anxiety Inventory & & \\
MBCT $\times 2$ months post & $-\mathbf{5 . 0 5}(1.86)$ & -8.72 to -1.38 \\
MBCT $\times 5$ months post & $-\mathbf{6 . 6 0}(1.89)$ & -10.33 to -2.87 \\
Psychoeducation $\times 2$ months post & $-\mathbf{4 . 8 6}(1.86)$ & -8.53 to -1.19 \\
Psychoeducation $\times 5$ months post & $-\mathbf{7 . 9 5}(1.81)$ & -11.52 to -4.37 \\
\hline Penn State Worry Questionnaire & & \\
MBCT $\times 2$ months post & $-1.99(1.95)$ & -5.85 to 1.86 \\
MBCT $\times 5$ months post & $-3.19(1.97)$ & -7.07 to 0.70 \\
Psychoeducation $\times 2$ months post & $-3.90(1.95)$ & -7.75 to -0.05 \\
Psychoeducation $\times 5$ months post & $-\mathbf{4 . 2 4}(1.89)$ & -7.98 to -0.51 \\
MBCT, mindfulness-based cognitive therapy. & & \\
a. Significant effects are in bold. & & \\
\hline
\end{tabular}

\section{Discussion}

Our study is the first that has compared MBCT with a low-intensity structured evidence-based intervention ${ }^{23}$ and a usual care control group. We had initially hypothesised that MBCT would be better than both psychoeducation and usual care in the reduction of anxiety and worry symptoms but this hypothesis was not supported. Our results showed that both MBCT and psychoeducation were better than usual care in the reduction of anxiety among people 
(a)

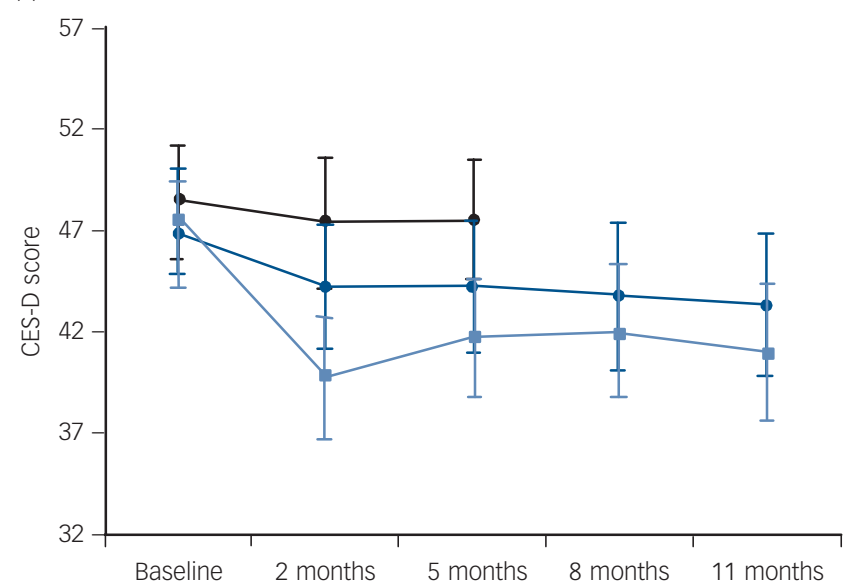

(b)

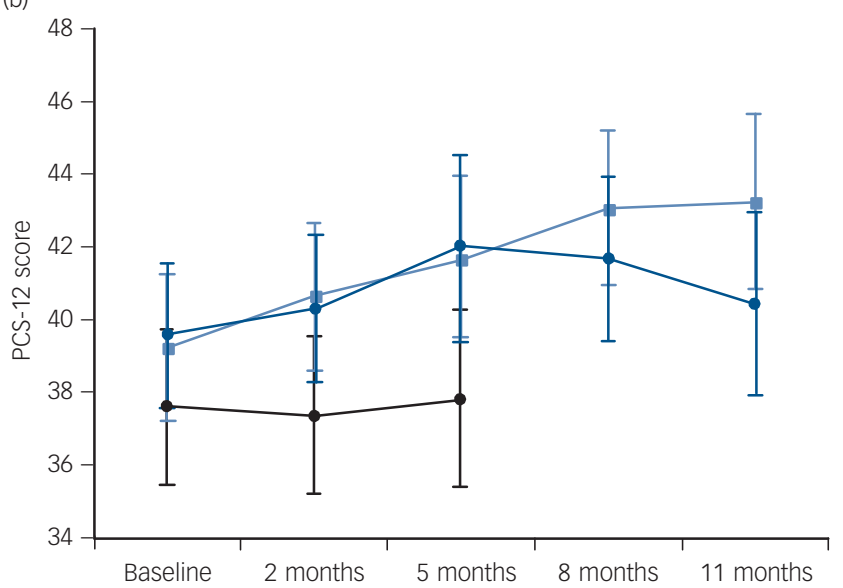

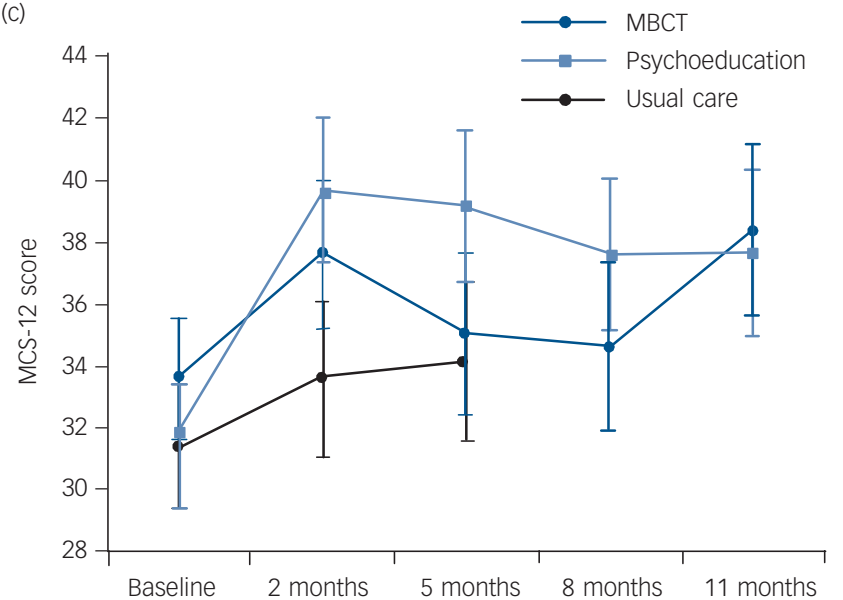

(d)

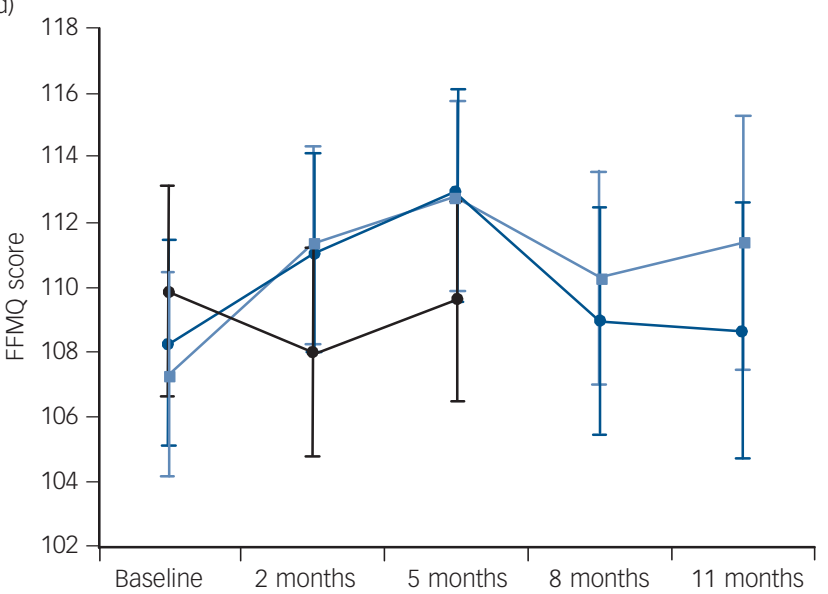

Fig. 3 Estimated mean scores for other outcome measures of participants in the mindfulness-based cognitive therapy (MBCT), psychoeducation and usual care groups over the study period.

(a) Depressive symptoms as measured by Center for Epidemiologic Studies Depression Scale (CES-D); (b) physical component of quality of life as measured by the Physical Component Summary of the Short Form-12 (PCS-12); (C) mental component of quality of life as measured by the Mental Component Summary of the SF-12 (MCS-12); (d) mindfulness as measured by the Five Facet Mindfulness Questionnaire (FFMQ). Error bars represent the $95 \%$ confidence intervals.

with GAD and that psychoeducation was better than usual care in helping to reduce worry symptoms, but only at the 5-month time point after baseline assessment. There was no statistically significant difference in primary outcomes between the psychoeducation and MBCT groups and we were unable to tell whether the lack of difference observed between these groups was statistically significant as the study was not powered to test for equivalence between the two interventions. However, the differences in outcomes between the MBCT and psychoeducation groups appear to be quite small and are likely to be of limited clinical significance. We also found that psychoeducation may have additional beneficial effects on the reduction of depressive symptoms and an improvement in mental health-related quality of life.

We are uncertain about the reasons for the superior effects of psychoeducation on worry and depressive symptoms, mental health-related quality of life as well as the higher attendance of participants in the psychoeducation group. We can speculate that culture-specific effects may have affected treatment expectancy and efficacy among patients. Although Hong Kong is a city and part of Asia with a long tradition of meditation practice (for example in Buddhism), the format and teaching of psychoeducation may be seen as a more scientific and modern Western approach when compared with that of MBCT (the majority of the contents of which is based on the teaching of meditation and can be perceived as similar to an ancient religious practice).

\section{Findings from other studies}

Although there are a lack of studies evaluating the efficacy of MBCT in reducing anxiety symptoms among people with GAD or comparing MBCT with group psychoeducation based on CBT principles, Koszycki and colleagues have compared the efficacy of 8-week MBSR, not MBCT, with a 12-week group CBT for patients with social anxiety disorder. ${ }^{43}$ They showed that although patients in both treatment groups improved in terms of anxiety symptoms, patients receiving the group CBT had a significantly larger reduction in their anxiety symptom scores, although the two interventions were similar in improving other aspects of mood, functionality and quality of life. More recently, Hoge et $a l^{22}$ have conducted an RCT evaluating the effects of MBSR, not MBCT, on anxiety and stress reactivity among people with GAD among 93 participants. They showed that MBSR was superior to an active control consisting of stress management didactic health education in reducing anxiety measured by both the BAI and Clinical Global Impression Scales. However, we must be cautious when comparing these findings with those of our study's since we have employed a different comparison group. We have used a didactic psychoeducation group using CBT principles plus relaxation skills training instead of a group CBT treatment as described by Koszycki et al $^{43}$ (group CBT assumed to be high intensity and psychoeducation assumed to be low intensity) or simple didactic presentations on stress management 
(assumed to have lower intensity since no CBT principles were introduced) as described by Hoge et al. ${ }^{22}$ The lack of observed superiority of a mindfulness intervention to psychoeducation as was shown in Hoge et al ${ }^{15}$ thus may have been because of the fact that our psychoeducation intervention may produce more therapeutic effects than the simple use of stress management education. Moreover, we also included simple relaxation skills, in the psychoeducation intervention, that may have added additional therapeutic effects. These differences in findings thus may make sense if we consider our psychoeducation group intervention to be a psychological intervention with lower intensity than that of a group CBT treatment, but of higher intensity than that of a stress management health education programme.

\section{Challenges measuring mindfulness}

Another unexpected findings of this study is that both the MBCT and psychoeducation groups showed improvements on 'mindfulness' as measured by the FFMQ. Although studies have been conducted to validate a number of scales to measure mindfulness, findings have been inconsistent. ${ }^{44}$ The current findings suggest that either the FFMQ is indeed not sensitive enough to measure the construct of mindfulness or that people who were randomised to the psychoeducation group did experience a change in their awareness or mindfulness as a result of the psychoeducation received, although the question of whether mindfulness can be measured using questionnaires remains. Furthermore, the main effect of group and time remained significant after adjusting for the FFMQ suggests that the FFMQ may not be a sensitive enough instrument to measure the positive changes associated with participation in a mindfulness intervention (results not shown).

\section{Limitations}

There are a number of limitations in our current study. First, there was much lower adherence in the MBCT group than the psychoeducation group. The mean number of sessions attended by participants randomised to the MBCT group was fewer than that of those randomised to the psychoeducation group. As a result, the attrition rate might have contributed to the smaller improvement on the other measured outcomes when compared with the participants randomised to the psychoeducation group, although our CACE analysis did not demonstrate any significant difference in outcomes because of adherence effect.

In this study, we initially aimed to recruit 228 participants with a presumed drop-out rate of $30 \%$ (159 participants) and we ended up recruiting 178 participants with 4 participants (2\%) dropping out of the study without any baseline data. At the end of the intervention, $55(90 \%), 54(89 \%)$ and $48(80 \%)$ questionnaires were collected in the MBCT, psychoeducation and usual care groups respectively, and a lower proportion of questionnaires were collected at the later time points. To account for the missing data because of non-returned or incomplete questionnaires, we used LMMs and have followed the intention-to-treat principle but the findings could have been affected by the incomplete data.

Third, our outcome measures were based on self-reported questionnaires collected at similar time points. Although all scales used in this study were validated, no clinician-rated instruments or diagnostic interviews were used at follow-up. As a result, we did not know whether the improvements in anxiety symptoms led to clinical remission of GAD.

Fourth, for ethical reasons we were only able to compare the two interventions (MBCT and psychoeducation) with the usual care group up to 3 months after treatment as it was unethical not to initiate treatment among these participants when the waiting time for psychiatric services was reduced. As a result, long-term findings were only available for the two intervention groups.

Fifth, we have included participants who at the time of recruitment had at least moderate levels of generalised anxiety symptoms, based on validated self-reported questionnaires, and the majority of participants were recruited via advertisements. As a result, we cannot generalise our results to patients who experience a milder degree of anxiety symptoms or to all patients in clinical settings and there may have been a selection bias of recruited participants being more motivated when compared with those patients seen in clinics.

Sixth, due to the design of our study, we were unable to tell whether differences between both the MBCT and psychoeducation interventions and usual care at 5 months were simply the result of differences in attention/time offered to participants rather than specific effects related to the content of either therapeutic modality, although there is established evidence that supports the effectiveness of psychoeducation for reducing anxiety symptoms.

Finally, we had two primary outcome measures and two comparisons in this study that could have caused a type I error because of multiple testing. However, our findings on performing Bonferroni correction (results not shown) show that the effects of the interventions on primary outcomes after correction remain largely the same.

\section{Implications}

This RCT showed that although participants from both the MBCT and psychoeducation groups had a significant decrease in anxiety symptoms as compared with the usual care group, psychoeducation appears to have greater acceptability and beneficial effects on worry symptoms and reduction in depressive symptoms among patients with GAD in this population. Future studies should be conducted to explore whether there are specific patient populations or unique patient characteristics that may be more suitable for either MBCT or group psychoeducation using CBT principles. In this respect, recent research has suggested that anxiety sensitivity ${ }^{45}$ or stress reactivity ${ }^{15}$ may differentially moderate treatment outcomes in CBT and adapted MBSR for anxiety disorders. ${ }^{45}$

\section{Samuel Yeung Shan Wong, MD, Benjamin Hon Kei Yip, PhD, Division of Family Medicine and Primary Care, Jockey Club School of Public Health and Primary Care, The Chinese University of Hong Kong, New Territories, Hong Kong; Winnie Wing Sze Mak, PhD, Department of Psychology, The Chinese University of Hong Kong, New Territories, Hong Kong; Stewart Mercer, MD, Institute of Health and Wellbeing, The University of Glasgow, Glasgow, UK; Eliza Yee Lai Cheung, PhD, Hong Kong Red Cross Headquarters, Hong Kong; Candy Yuet Man Ling, MSSC, New Life Psychiatric Rehabilitation Association, Kowloon, Hong Kong. Wacy Wai Sze Lui, MSSC, Hospital Authority, Kow Authorty, Kowloon, Hong Kong: Wai Kwong Tang, MD, Department of Pychiatry, The Chinese University of Hong Kong, New Territories, Hong Kong; Herman Hay
Ming Lo, PhD, Department of Applied Social Studies, The City University of Hong Kong, Hong Kong; Justin Che Yuen Wu, MD, Institute of Integrative Medicine, Faculty of Medicine, The Chinese University of Hong Kong, New Territories, Hong Kong; Tatia Mei Chun Lee, PhD, Department of Psychiatry and Department of Medicine, The University of Hong Kong, Hong Kong Island, Hong Kong; Ting Gao, MSc, Sian M. Griffiths, MSc, Division of Family Medicine and Primary Care, Jockey Club School of Public Health and Primary Care, The Chinese University of Hong Kong, New Territories, Hong Kong; Peter Hoi Sing Chan, MSSC, Holy Trinity Bradbury Centre, Kowloon City, Kowloon, Hong Kong; Helen Shuk Wah Ma, PhD, Centre of Buddhist Studies, The University of Hong Kong, Hong Kong Island, Hong Kong, China}

Correspondence: Samuel Yeung Shan Wong, 4/F, School of Public Health, Prince of Wales Hospital, Shatin, New Territories, Hong Kong SAR, China. Email: yeungshanwong@cuhk.edu.hk

First received 17 Jun 2014, final revision 3 Mar 2015, accepted 14 May 2015

\section{Funding}

This study was funded by the Health and Health Services Research Fund of the Food and Health Bureau of the HKSAR government with grant reference number 07080451. The funders had no role in study design, data collection and analysis, decision to publish or preparation of the manuscript. 


\section{Acknowledgements}

We would also like to thank all the participants, clinicians and healthcare workers who have contributed to patient recruitment in this study.

\section{References}

1 American Psychiatric Association. Diagnostic and Statistical Manual of Mental Disorders (4th edn) (DSM-IV). APA, 1994.

2 Kessler RC, Walters EE, Wittchen HU. Epidemiology. In Generalized Anxiety Disorder: Advances in Research and Practice (eds RG Heimberg, CK Turk DS Mennin): 29-50. Guilford Press, 2004.

3 Greenberg PE, Sisitsky TK, Kessler RC, Finkelstein SN, Berndt ER, Davidson JRT, et al. The economic burden of the anxiety disorders in the 1990s. J Clin Psychiatry 1999; 60: 427-35.

4 Cuijpers P, Sijbrandij M, Koole S, Huibers M, Berking M, Andersson G. Psychological treatment of generalized anxiety disorder: a meta-analysis. Clin Psychol Rev 2014; 34: 130-40.

5 Waters AM, Craske MG. Generalized anxiety disorder. In Improving Outcomes and Preventive Relapse in Cognitive Behavioural Therapy (eds MM Antony, DR Ledley, RG Heimberg): 77-127. Guilford, 2005.

6 Sundquist J, Lilja A, Palmer KM, Menon AA, Wang X, Johansson LM, et al. Mindfulness group therapy in primary care patients with depression, anxiety and stress and adjustment disorders: randomized controlled trial. Br J Psychiatry 2015; 206: 128-35.

7 Kabat-Zinn J. Full Catastrophe Living: Using the Wisdom of your Body and Mind to Face Stress, Pain, and Illness. Delacourt, 1990.

8 Chiesa A, Serretti A. Mindfulness-based interventions for chronic pain: a systematic review of the evidence. J Altern Complement Med 2011; 17: 83-93.

9 Chiesa A, Serretti, A. Mindfulness-based stress reduction for stress management in healthy people: a review and meta-analysis. J Altern Compl Med 2009; 15: 593-600.

10 Ledesma $\mathrm{D}$, Kumano $\mathrm{H}$. Mindfulness based stress reduction and cancer: a meta-analysis. Psychooncology 2009; 18: 571-9.

11 Fjorback LO, Arendt $M$, Ørnbøl E, Fink $P$, Walach $H$. Mindfulness-based stress reduction and mindfulness-based cognitive therapy? A systematic review of randomized controlled trials. Acta Psychiatr Scand 2011; 124: 102-9.

12 Segal ZV, William JM, Teasdale JD. Mindfulness-Based Cognitive Therapy for Depression. A New Approach to Preventing Relapse. Guilford Press, 2002.

13 Chiesa A, Serretti A. Mindfulness based cognitive therapy for psychiatric disorders: a systematic review and meta-analysis. Psychiatry Res 2011; 187 441-53

14 Williams JMG, Kuyken W. Mindfulness-based cognitive therapy: a promising new approach to preventing depressive relapse. Br J Psychiatry 2012; 200 $359-60$

15 Evans S, Ferrando S, Findler M, Stowell C, Smart C, Haglin D. Mindfulnessbased cognitive therapy for generalized anxiety disorder. J Anxiety Disord 2008; 22: 716-21.

16 Craigie MA, Rees CS, Marsh A, Nathan P. Mindfulness-based cognitive therapy for generalized anxiety disorder: a preliminary evaluation. Behav Cogn Psychother 2008; 36: 553-68.

17 Yook K, Lee SH, Ryu M, Kim KH, Choi TK, Suh SY, et al. Usefulness of mindfulness-based cognitive therapy for treating insomnia in patients with anxiety disorder: a pilot study. J Nerv Ment Dis 2008; 196: $501-3$.

18 Vollestad J, Nielsen MB, Neilsen GH. Mindfulness and acceptance-based interventions for anxiety disorders: a systematic review and meta-analysis. Br J Clin Psychol 2012; 51: 239-60.

19 Kim YW, Lee SH, Choi TK, Suh SY, Kim B, Kim CM, et al. Effectiveness of mindfulness-based cognitive therapy as an adjuvant to pharmacotherapy in patients with panic disorder or generalized anxiety disorder. Depress Anxiety 2009; 26: 601-6.

20 Kabat-Zinn J, Massion AO, Kristeller J, Peterson LG, Fletcher KE, Pbert L, et al. Effectiveness of a meditation-based stress reduction program in the treatment of anxiety disorders. Am J Psychiatry 1992; 149: 936-43.

21 Vøllestad J, Sivertsen B, Nielsen GH. Mindfulness based stress reduction for patient with anxiety disorders: evaluation in a randomized controlled trial. Behav Res Ther 2011; 49: 281-8.

22 Hoge EA, Bui E, Marques L, Metcalf CA, Morris LK, Robinaugh DJ, et al. Randomized controlled trial of mindfulness meditation for generalized anxiety disorder: effects on anxiety and stress reactivity. J Clin Psychiatry 2013; 74: 786-92.

23 National Institute for Health and Care Excellence. Anxiety (amended). Management of Anxiety (Panic Disorder, with or without Agoraphobia, and Generalised Anxiety Disorder) in Adults in Primary, Secondary and Community Care. NICE Clinical Guidance 22 (amended). NICE, 2007 (https:// www.jpshealthnet.org/sites/default/files/anxiety_disorder_pdq.pdf).

24 Wong SYS, Mak WWS, Cheung ELY, Ling CY, Lui WW, Tang WK, et al. A randomized, controlled clinical trial: the effect of mindfulness-based cognitive therapy on generalized anxiety disorder among Chinese community patients: protocol for a randomized trial. BMC Psychiatry 2011; 11: 187

25 Kam IWK. Development of the Bilingual (Chinese/English) SCID-I (Structured Clinical Interview for DSM-IV Axis I disorder): A Study of its Reliability and Validity in an In-Patient Population. Dissertation for Part III Examination of Fellowship. Hong Kong College of Psychiatrists, 2000.

26 Cheng SKW, Wong CS, Wong KC, Chong GS, Wong M, Chang SS, et al. A study of psychometric properties, normative scores, and factor structure of the Beck Anxiety Inventory - the Chinese version. Chinese J Clin Psychol 2002; 10: 4-6

27 Spiter RL, Kroenke K, William JB. Validation and utility of a self-report version of PRIME-MD: the PHQ primary care study. Primary Care Evaluation of Mental Disorders. Patient Health Questionnaire. JAMA 1999; 282: 1737-44.

28 White J. Treating Anxiety and Stress: A Group Psycho-Educational Approach using Brief CBT. John Wiley \& Sons, 2000.

29 Beck AT, Epstein N, Brown G, Steer RA. An inventory for measuring clinical anxiety: psychometric properties. J Consult Clin Psychol 1988; 56: 893-7.

30 Beck AT, Steer RA. Beck Anxiety Inventory Manual. Psychological Corporation, 1990

31 Meyer TJ, Miller ML, Metzger RL, Borkovec TD. Development and validation of the Penn State Worry Questionnaire. Behav Res Ther 1990; 28: $487-95$

32 Zhong J, Wang C, Li J, Liu J. Penn State Worry Questionnaire: structure and psychometric Properties of the Chinese version. J Zhejiang Univ Sci B 2009; 10: 211-8.

33 Cheung $\mathrm{CK}$, Bagley C. Validating an American scale in Hong Kong: The Centre of Epidemiological Studies depression scale (CES-D). J Psychol 1998; 132 169-86.

34 Lam CLK, Tse EYY, Gandek B. Is the standard SF-12 health survey valid and equivalent for a Chinese population. Qual Life Res 2005; 14: 539-47.

35 Baer RA, Smith GT, Hopkins J, Krietemeyer J, Toney L. Using self-report assessment methods to explore facets of mindfulness. Assessment 2006; 13 27-45.

36 Hou J, Wong SYS, Lo HHM, Mak WWS, Ma HSW. Validation of a Chinese version of the Five Facet Mindfulness Questionnaire in Hong Kong and development of a short form. Assessment 2014; 21: 363-71.

37 Dunn G, Maracy M, Dowrick C, Ayuso-Mateos JL, Dalgard OS, Page H et al. Estimating psychological treatment effects from a randomised controlled trial with both non-compliance and loss to follow-up. $\mathrm{Br}$ Psychiatry 2003; 183: 323-31.

38 Angrist JD, Imbens GW, Rubin DB. Identification of causal effects using instrumental variables (with discussion). J Am Stat Assoc 1996; 91: 444-72.

39 Frangakis CE, Robin DB. Addressing complications of intention-to-treat analysis in the combined presence of all-or-none treatment-noncompliance and subsequent missing outcomes. Biometrika 1999; 86: 365-79.

40 Hernán MA, Hernández-Díaz S. Beyond the intention-to-treat in comparative effectiveness research. Clin Trials 2012; 9: 48-55.

41 Dodd S, White IR, Williamson P. Nonadherence to treatment protocol in published randomized controlled trials: a review. Trials 2012; 13: 84

42 Wetherell JL, Gats M, Craske MG. Treatment of generalized anxiety disorder in older adults. J Consult Clin Psychol 2003; 71: 31-40.

43 Koszycki D, Benger M, Shlik J, Bradwejn J. Randomzied trial of a meditationbased stress reduction program and cognitive behavior therapy in generalized social anxiety disorder. Behav Res Ther 2007; 45: 2518-26.

44 Park T, Reilly-Spong M, Gross CR. Mindfulness: a systematic review of instruments to measure an emergent patient-reported outcome (PRO). Qual Life Res 2013; 22: 2639-59.

45 Arch JJ, Ayers CR. Which treatment worked better for whom? Moderators of group cognitive behavioral therapy versus adapted mindfulness based stress reduction for anxiety disorders. Behav Res Ther 2013; 51: 434-42. 